\title{
Huez, Oz, Vaujany, Saint-Sorlin-d'Arves
}

Prospections dans le massif des Rousses

Joël Vital

\section{(2) OpenEdition}

Édition électronique

URL : http://journals.openedition.org/adlfi/3493

ISSN : 2114-0502

Éditeur

Ministère de la culture

Référence électronique

Joël Vital, « Huez, Oz, Vaujany, Saint-Sorlin-d'Arves », ADLFI. Archéologie de la France - Informations [En ligne], Rhône-Alpes, mis en ligne le 01 mars 2009, consulté le 01 mai 2019. URL : http:// journals.openedition.org/adlfi/3493

Ce document a été généré automatiquement le 1 mai 2019.

(c) Ministère de la Culture et de la Communication, CNRS 


\title{
Huez, Oz, Vaujany, Saint-Sorlin- d'Arves
}

\author{
Prospections dans le massif des Rousses
}

\section{Joël Vital}

\section{Identifiant de l'opération archéologique : 9970}

Date de l'opération : 2009 (PT)

1 Faisant suite à deux précédentes campagnes de prospection thématique en 2007 et 2008, les opérations conduites en 2009 avaient plusieurs buts :

2 - d'un point de vue général, la poursuite des reconnaissances gitologiques et minéralogiques associée à la cartographie des recouvrements et l'évolution géomorphologique ;

3 - pour le secteur minier prospecté antérieurement en Isère (2000 m-2700 m), l'extension des recherches au sud, en direction des zones d'exploitations d'âge médiéval, les opérations de la fin de campagne 2008 ayant révélé de nouveaux travaux extractifs de typologie protohistorique vers le Dôme des Rousses et l'Alpe d'Huez; ce volet visait simultanément à l'identification des zones de passages entre les gradins des $2000 \mathrm{~m}$ et des $2600 \mathrm{~m}$;

- l'extension des prospections au nord, côté Savoie, dans le secteur du lac Bramant dans lequel un carottage sédimentaire avait révélé une pollution au cuivre et au zinc datée du Bronze ancien ; cette zone n'avait pas encore fait l'objet de prospection ;

- cette extension géographique visait ainsi à définir les limites du champ minier ;

6 - en règle générale, une évaluation de surface et par tariérage des potentialités de compréhension des conditions paléoenvironnementales dans des zones de sédimentation, notamment des zones humides ;

$7 \quad$ - le démarrage des échantillonnages palynologiques. 
8 La campagne s'est déroulée sur 3 semaines au mois d'août avec une équipe de 3 à 4 personnes, en 3 étapes.

9 La première étape a été consacrée aux secteurs du gradin 2500 m-2600 m qui font suite vers le sud à la zone où se concentrent la majorité et les plus importants travaux miniers du Bronze ancien. Plusieurs nouveaux filons testés localement par des tailles au feu ont été inventoriés dans le secteur de Balme Rousse. Plus au sud, entre les secteurs des lacs de La Fare et du Milieu, jusqu'au Dôme des Rousses, nous pouvons souligner l'absence de traces minières métallurgiques des âges de Métaux. Par contre, un dense réseau filonien a été topographié, mais les minéralisations semblaient insuffisantes pour justifier des travaux. Par contre, des cristallisations de quartz automorphes ont été identifiées à l'ouest de cette vaste zone, en pied de falaise, au sein d'importantes excavations, formant abris et grotte, réalisées aux dépens de diaclases ouvertes subhorizontales. Deux nouvelles structures, de formes différentes, ont par contre été localisées incidemment lors de nos traversées des zones de Balme Rousse et du Plan des Cavalles parcourues antérieurement (2007-2008). Il s'agit d'une série de murs à angles droits et d'une structure ovalaire à pierres parfois de chant, proches des tranchées minières du Plan des Cavalles 1 et 2 . Au Plan des Cavalles 10, de nouvelles cupules de test au feu implantées sur des filons minéralisés ont été découvertes. Les divers cheminements que nous avons empruntés indiquent que l'accès entre les différents gradins s'opère en plusieurs points sans difficulté majeure.

10 La deuxième étape a consisté à prolonger notre prospection sur le même palier, vers le nord cette fois. Dans le secteur du col du Sabot, une vaste aire où se concentrent des minéralisations de quartz automorphe a été localisée. Elle fait l'objet d'une intense et dégradante exploitation moderne en tranchées. Un second volet a été consacré aux prélèvements palynologiques pour lesquels nous avons prêté notre concours à F. David (CEREGE). Deux carottes ont été extraites côté Isère, au sud de la zone de l'Alpette. L'une, au sud du lac Carrelet (ALP1, alt. $2030 \mathrm{~m}$ ) d'une puissance exceptionnelle de $4,50 \mathrm{~m}$ et couvrant potentiellement les 9 derniers millénaires, riche en macrorestes échantillonnés pour la construction d'un modèle d'âge radiocarbone. La seconde, en contrehaut immédiat du lac Faucille (FAU 1, alt. $2100 \mathrm{~m}$ ), d'une puissance de 1,70 m, couvrirait la fin de l'Holocène.

11 La troisième période de prospections s'est déplacée sur la Savoie, entre le Dôme de la Cochette et le Col de la Croix de Fer, dans la tranche altitudinale $2400 \mathrm{~m}-2700 \mathrm{~m}$. Il s'agissait de définir au nord les limites du champ minier à partir d'une vaste zone qui n'avait jusqu'alors fait l'objet d'aucune prospection, mais potentiellement intéressante du fait de l'enregistrement de paléopollutions métalliques dans le lac Bramant. Nous avons observé une répartition particulière des systèmes filoniens et des zones minéralisées, avec des réseaux de filons de taille et de densité importantes; les concentrations minérales se succèdent surtout selon un axe sud-sud-ouest - nord-nord-est sur le tombant rocheux qui limite et domine à l'ouest les lacs. Les travaux miniers n'ont pas été datés pour l'heure, mais les modes des exploitations par le feu est conforme à celles connues côté Isère. Les formes des exploitations sont comparables, qu'il s'agisse des cuvettes d'évaluation depuis le sol, des attaques obliques en courtes tranchées orientées en profondeur, certaines noyées, des tests depuis les pieds de parois, et enfin des chapelets de cavités allongées et alignées des mines les plus importantes. Celles-ci sont d'ailleurs accompagnées de haldes, de quelques zones de concassage et de rares structures en élévation. Par contre, aucune réalisation de l'ampleur de celles du Balme Rousse 2 ou du 
Plan des Cavalles 4 n'a été découverte. Sous le pointement rocheux sur lequel est édifié le refuge de l'Etendard, qui nous servait de base, plusieurs constructions de pierres sèches, cellules, enclos, associées à des blocs volumineux pris dans un large cône d'éboulis, ont été inventoriées, qui suggèrent une activité pastorale non datée. Ces vestiges ont été globalement peu nombreux cette année, notre progression s'étant faite essentiellement dans un paysage très minéral.

Les perspectives ouvertes pour une dernière campagne de terrain en 2010 concernent en premier lieu la fin des prospections pédestres au nord de la zone métamorphisée, proche du col de la Croix de Fer. Un second volet verra le prélèvement de nouvelles carottes destinées à la recherche palynologique et des fossiles non polliniques, ainsi que la caractérisation plus fine des pollutions anciennes, comme des processus de sédimentation qui ont permis leur enregistrement.

13 Moulin Bernard, Thirault Éric et Vital Joël

\section{INDEX}

operation Prospection thématique (PRT)

Thèmes : feu, mine, paléoenvironnement, quartz, taille

Index géographique : Rhône-Alpes, Isère (38), Huez

\section{AUTEUR}

JOËL VITAL

CNRS 